\title{
Histerectomia Vaginal ou colpocleise de Le Fort em pacientes idosas com prolapso uterino total: uma revisão narrativa
}

\author{
Vaginal hysterectomy or Le Fort colpocleise in elderly patients with total uterine prolapse: \\ a narrative review
}
Histerectomía vaginal o colpocleise de Le Fort en pacientes de edad avanzada con prolapso uterino total: una revisión narrativa

Suelen Carvalho de Araújo ${ }^{1 *}$, Luis Alberto Leon Camac.

\begin{abstract}
RESUMO
Objetivo: Descrever sobre histerectomia vaginal ou colpocleise de Le Fort em pacientes idosas com prolapso uterino total, evidenciando seus benefícios e suas desvantagens. Revisão Bibliográfica: Em relação aos benefícios, recorrentemente se promulga que a histerectomia vaginal compreende uma via segura de tratamento, já a Colpocleise ou operação de Le Fort aborda um técnica cirúrgica obliterativa utilizada no tratamento do grande prolapso uterino e/ou de cúpula vaginal sintomáticos, em pacientes que não mais desejam ter vida sexual, de idade avançada e risco cirúrgico elevado. Considerações Finais: Mesmo que algumas mulheres portadoras de prolapso uterino completo obstruam sua atividade sexual devido à sua condição clínica, muitas ainda mostram o desejo de reiniciar a vida, mesmo atingindo quando atingem a terceira idade, buscando valorizar a função sexual, sendo de extrema importância o desenvolvimento de técnicas cirúrgicas que protejam a anatomia e função vaginal, que sejam seguras para as pacientes e portadoras de comorbidades. As vantagens benéficas que a histerectomia vaginal traz promove que é o melhor procedimento a ser realizado, pois dispoem de menor perda sanguínea intra-operatória, diminuição da intensidade de dor pós-operatória, baixa freqüência de utilização de analgésicos e menor tempo de retorno às atividades após a cirurgia.
\end{abstract}

Palavras-chave: Histerectomia vaginal, Cirurgia, Prolapso uterino.

\begin{abstract}
Objective: To describe Le Fort vaginal hysterectomy or colpocleisis in elderly patients with total uterine prolapse, highlighting its benefits and disadvantages. Bibliographic review: In relation to the benefits, it is often promulgated that vaginal hysterectomy comprises a safe treatment route, whereas Colpocleisis or Le operation Fort addresses an obliterative surgical technique used in the treatment of symptomatic large uterine prolapse and / or vaginal dome, in patients who no longer wish to have sex, old age and high surgical risk. Final Considerations: Even if some women with uterine prolapse obstruct their sexual activity due to their clinical condition, many still show the desire to restart life, even reaching when they reach old age, seeking to enhance sexual function, and the development of surgical techniques that protect anatomy and function is extremely important. the vaginal, which are safe for patients and patients with comorbidities. The beneficial advantages that vaginal hysterectomy brings promote that it is the best procedure to be performed, as they have less intraoperative blood loss, decreased postoperative pain intensity, low frequency of use of analgesics and less time to return to activities after surgery.
\end{abstract}

Keywords: Hysterectomy vaginal, Surgery, Uterine prolapse.

${ }^{1}$ Universidade Federal do Amazonas (UFAM). Manaus - AM. *E-mail: suelen25araujo@outlook.com

SUBMETIDO EM: 2/2020

ACEITO EM: 3/2020

PUBLICADO EM: 4/2020

REAS/EJCH | Vol.Sup.n.47 | e3105 | DOI: https://doi.org/10.25248/reas.e3105.2020 Página 1 de 8 


\section{RESUMEN}

Objetivo: Describir la histerectomía vaginal o la colpocleisis de Le Fort en pacientes de edad avanzada con prolapso uterino total, destacando sus beneficios y desventajas. Revisión bibliográfica: En relación con los beneficios, a menudo se promulga que la histerectomía vaginal comprende una ruta de tratamiento segura, mientras que la colpocleisis o la operación Le Fort aborda una técnica quirúrgica obliterante utilizada en el tratamiento del prolapso uterino grande sintomático y / o la cúpula vaginal, en pacientes que ya no desean tener relaciones sexuales, vejez y alto riesgo quirúrgico. Consideraciones finales: Incluso si algunas mujeres con prolapso uterino obstruyen su actividad sexual debido a su condición clínica, muchos todavía muestran el deseo de reiniciar la vida, incluso cuando alcanzan la vejez, buscando mejorar la función sexual, y el desarrollo de técnicas quirúrgicas que protegen la anatomía y la función es extremadamente importante. la vaginal, que es segura para pacientes y pacientes con comorbilidades. Las ventajas beneficiosas que brinda la histerectomía vaginal promueven que es el mejor procedimiento a realizar, ya que tienen menos pérdida de sangre intraoperatoria, disminución de la intensidad del dolor postoperatorio, baja frecuencia de uso de analgésicos y menos tiempo para volver a las actividades despues de la cirugia.

Palabras clave: Histerectomía vaginal, Cirugía, Prolapso uterino.

\section{INTRODUÇÃO}

O prolapso genital é uma patologia que atinge grande parte populacional feminina. Evidência que cerca de $30-50 \%$ das mulheres desenvolve o risco durante a sua vida ter um prolapso de órgão pélvico, sendo que apenas $2 \%$ destas mulheres tornam-se sintomáticas (SAMUELSSON EC, et al., 1999). É mais adequado a realização da cirurgia para mulheres que estão em maior risco cirúrgico devido a comorbidades médicas e não estão pensando em futuro coito vaginal (FITZGERALD MP, et al., 2008).

Infecções são considerada a complicação mais comum na histerectomia vaginal (HV) sua variação pode ocorrer entre 1 a 5\%. Multifatores estão alistados à infecção, podendo ser relacionados a: manipulação excessiva dos tecidos, comorbidades que diminuem a imunidade, tempo cirúrgico prolongado, vulvo-vaginites prévias, e principalmente a formação de hematomas (SEGEV Y, et al., 2010).

A incidência do prolapso vaginal aumenta com o envelhecimento, não sendo raro encontrar mulheres idosas com prolapso vaginal sintomático. A ocorrência e predomínio do prolapso dos órgãos pélvicos, de acordo com a Sociedade Internacional de Continência (ISC) conseguem variar de 2,9\% a 11,4\%. É um método de técnica para abordagem inovadora de Le Fort que consiste em acreditar que a disfunção do pavimento pélvico proveniente do parto, acometia o "afundamento" da parede vaginal antecedente, que quando feito por "arrastamento" condicionaria o mesmo efeito à restante vagina. Sendo assim, ponderou que a prevenção do prolapso uterino seria plausível, se conservar a agregação das paredes da vagina. Observou-se ainda, que o hiato vaginal extenso, traz contribuição para o ocorrer acidentes durante o procedimento cirúrgico (FITZGERALD MP, et al., 2006).

Bases histórica sobre a histerectomia foram relatadas 120 depois de Cristo (d.C), onde teve a primeira realização por via vaginal na Grécia por Soranus a fim de tratar um prolapso uterino (SUTTON C, 1997). A partir deste momento, somente na Idade Média vai se relatar novos procedimentos vaginais, grande parte no pós-parto. Hystera consiste na definição da palavra de origem grega que expressão significado de útero, sendo este um órgão feminino que constitui um formato de pera, de fundamental importância para o ciclo reprodutivo humano, uma vez que é no útero que se realiza todo o desenvolvimento embrionário (MELO MCB e BARROS EN, 2009).

Histerectomias são realizadas por vias sendo ela a abdominal, vaginal ou cirurgia minimamente invasiva (com ou sem a assistência robótica). Diversos fatores podem influenciar qual será a via da histerectomia, tais como o tamanho e forma da vagina e do útero, a acessibilidade, presença de doença extrauterina, precisão de procedimentos concomitantes, treinamento e experiência do cirurgião, quais tecnologias estão disponíveis no hospital, recursos, e em muitos casos a preferência do paciente (JACOBY VL, et al., 2009). 
Heisler CA, et al. (2009) discorre da forma que como em todo procedimento médico, a HV tem a possível complicação por possuir diversas variáveis que podem desempenhar a interferência na incidência, uma delas destacada como comorbidades. A histerectomia é considerada a segunda cirurgia ginecológica mais frequentemente realizada nos países desenvolvidos em mulheres em idade reprodutiva, ficando atrás apenas da cesariana (WU JM, 2007).

A seleção do tipo de correção cirúrgica depende dos multifatores tais como: faixa etária , as queixas urinárias, o desejo ou não de acondicionar a função vaginal, da comorbilidade, que intervém com o risco durante o procedimento cirúrgico ou da anestesia e das falhas vigentes no pavimento pélvico (MAHERCF, et al., 2007). O tratamento cirúrgico do prolapso do órgão pélvico envolve procedimentos reconstrutivas e obliterante, os procedimentos obliterante, embora menos freqüentemente realizada, são um instrumento importante para os cirurgiões pélvicos, tendo em conta a elevada eficácia e baixa morbidade (NÍTIDO CC, et al., 2013).

Algumas das indicações mais comuns para a histerectomia são condições que têm uma tendência para uma maior útero ou adesões, tais como leiomioma (51,4\%), hemorragia uterina anormal $(41,7 \%)$, e endometriose (30\%). Estas condições podem tornar-se HV mais tecnicamente difícil em muitos pacientes. Como uma das principais barreiras citadas para realização de HV é dificuldades técnicas (EINARSSON Jl, et al., 2010).

A capacidade de operar por via vaginal é uma características que distingue a cirurgia ginecológica de outras especialidades. Sendo a histerectomia indicada de forma quase exclusiva para tratamento de prolapso uterino. Piazza MJ, et al. (2010), ressalta que a prática de histerectomia se refere a um dos procedimentos cirúrgicos mais frequentes em execução na atualidade, especialmente pelo fato de ser uma intervenção cirúrgica ginecológica indicada para o tratamento de diversas doenças.

Colpocleise ou operação de Le Fort: é uma técnica cirúrgica obliterativa utilizada no tratamento em casos que há grande prolapso uterino, ou apresente cúpula vaginal sintomáticos, em pacientes que optam por não mais ter atividade sexual, ou já estarem em idade avançada e com predisposição a risco cirúrgico superior. O que ocorre é a retirada de uma parte da faixa da mucosa vaginal anterior e outra da posterior. Em seguida, faz-se à sutura da parede anterior com a parede posterior da vagina, extinguindo uma parte o lúmen, o que faz a criação de um septo largo entre as paredes anterior e posterior da vagina, adequando a sustentação uterina e da cúpula vaginal (SCHORGE JO, 2011).

A cirurgia obliterativa traz como vantagem comparativa à colporraia, notadamente no caso da colpoclese total, um percentual de sucesso independentemente do tamanho do prolapso e do defeito anatômico (MAHER CF, et al., 2007). Fazendo a comparação com a sacrocolpopexia e a suspensão ao ligamento sacroespinhoso, a colpoclese total tem melhor aplicabilidade, é altamente improvável o acometimento de lesões noutros órgão.

É uma metodologia usada e aplicada apenas em mulheres na pós-menopausa com prolapso uterino total. É importante e necessário que a cérvice e a cavidade endometrial sejam completamente avaliadas por meio do esfregaço citológico e por dilatação e curetagem afim de trazer a garantia que não haja evidências de neoplasia maligna uterina escondida antes do fechamento da vagina. Para a realização do procedimento é necessário que a paciente fique em posição de litotomia, nesse momento puxa-se a cérvice para a parte exterior com o auxílio do tenáculo. No deslocamento uterino total, a exposição da cérvice para parte externa everte quase completamente a vagina (WALLWIENER D, 2012).

Menard JP (2008), em um dos seus estudos reafirma que os bons resultados da colpocleise depender de variáveis dentre elas: Repassar a informação ao paciente sobre os benefícios e possíveis complicações que a técnica pode oferecer, além de ser importante a orientação das famílias, já que na maioria deste casos os pacientes são mais velhos e em muitos casos dependentes. Promover a avaliação completa do colo do útero e endométrio, e do tratamento de escaras no pré-operatório que possa existir. Avaliar o estado clínico, em virtude de prevenir de infecção, tromboembolismo e outras comorbidades. Os questionários de qualidade de vida, urinário e sintomas digestivos, quantificação de prolapso do POP-Q, é obrigatória se a participação em instituições acadêmicas. Regulamentação técnica, preservando a mucosa suburetral (MENARD JP, 2008). 
Desta forma o presente trabalho realizado por revisão da literatura teve por objetivo descrever através de uma revisão bibliográfica sobre HV ou colpocleise de Le Fort em pacientes idosas com prolapso uterino total, evidenciando seus benefícios e suas desvantagens.

\section{REVISÃO BIBLIOGRÁFICA}

\section{Conceito e definição de histerectomia vaginal}

No que entende por benefícios, no cotidiano se relata que a histerectomia vaginal alcança a ser uma via segura e simples, dando a competência aos ginecologistas, de forma geral, instigar estímulos ao desenvolvimento e à prática do procedimento, muito conhecida de "histerectomia por orifício natural" ou de "histerectomia sem cicatriz visível" (METTLE RL, et al., 2010).

Desta maneira, de forma geral, atenta-se que a via vaginal proporciona menos trauma cirúrgico para o paciente, ocasionando a recuperação da vida cotidiana e do ritmo intestinal mais rápido (ADORNO SDS, et al., 2018). O autor Figueiredo O (1999), já ressalta o aumento no número de histerectomia com o passar dos anos como indicações da cirurgia vaginal. Desvantagens interligadas a histerectomia versam na dissecção da fáscia endopélvica e secção dos ligamentos útero-sacros e cardinais, o que acometerá o processo de cicatrização em relação a perda da qualidade do tecido fibromuscular (CARRAMÃO $S$, et al., 2009).

Lima JM, et al. (2013), relatou que durante os meses de março de 2011 a março de 2013, 19 mulheres idosas foram admitidas no hospital estudado para serem submetidas à HV, onde a indicação foi devido ao prolapso uterino. A idade das mulheres que realizaram a cirurgia se dá da seguinte forma: 5 possuíam idade entre 60 a 64 anos (26\%), 5 entre 65 a $69(26 \%), 0$ entre 70 a $74(0 \%), 5$ entre 75 a $79(26 \%), 3$ entre 80 a $84(16 \%), 1$ entre 85 a 89 (5\%). Com relação à paridade tem-se: 1 mulher apresentou 1 paridade (5\%), 3 deram 2 paridades (16\%), 1 apresentou 3 paridades (5\%), 1 apresentou 4 paridades (5\%), 1 apresentou 05 paridades (5\%), 1 apresentou 6 paridades (5\%), 0 apresentou 7 paridade $(0 \%), 1$ apresentou 8 paridades (5\%), 3 apresentaram 9 paridades (16\%), 2 apresentaram 10 paridades (11\%), 0 apresentou 11 paridades $(0 \%), 2$ apresentaram 12 paridades (11\%), 0 apresentou 13 paridades $(0 \%), 1$ apresentou 14 paridades $(5 \%)$, 0 apresentou 15 paridades (0\%), 0 apresentou 16 paridades $(0 \%), 1$ apresentou 17 paridades $(5 \%), 1$ não foi notificada (5\%).

Os resultados do estudo de Costa AAR, (2003), sugeriram que a histerectomia total, efetivada pela via vaginal para pacientes sem prolapso uterino com designação cirúrgica por doenças benignas, formam uma abordagem cirúrgica com melhores resultados e diminui o nível de complicações intra e pós operatórias. Evidenciou-se que a quantidade de sangue perdido durante o ato operatório foi expressivamente menor no grupo de mulheres sujeitadas à $\mathrm{HV}$ em relação à cirurgia abdominal, com uma tendência a menor freqüência de hemotransfusão pós-operatória. A intensidade da dor e a utilização de analgésicos nas doze horas seguintes ao procedimento também foram significativamente menores no grupo da HV. Não houve diferenças significativas em relação ao tempo de cirurgia, ao tempo de anestesia e à permanência hospitalar. Por fim, o retorno às atividades habituais foi mais precoce nas pacientes submetidas à histerectomia total pela técnica vaginal.

Em um estudo de coorte prospectivo realizado nos Estados Unidos, analisaram-se 1.427 mulheres submetidas à histerectomia total, por via vaginal, vaginal assistida por laparoscopia ou abdominal, todas com doenças benignas confinadas ao útero e volume uterino menor que $280 \mathrm{~g}$. Observaram-se diversas vantagens para a via vaginal, destacando-se a menor média no tempo de permanência hospitalar (3,9 contra 2,7 dias, $p$ $<0,001$ ) e uma redução significativa do risco de complicações, com uma freqüência de $9,3 \%$ para as abdominais e de $5,3 \%$ para as vaginais $(p<00,1)$ (KOVAC SR, 2000).

No estudo de Maher CF (2001), a operação de colpocleise a le fort falhou em três dos 27 pacientes. Duas falhas ocorreram nos primeiros 3 meses após a cirurgia, e outro em 1 ano. A nossa taxa de falhas de 11\% está em conformidade com a de 2,4-27\% para fixação sacroespinhal e 1,3-16\% para sacrocolpopexy (MARCUS CP, et al., 2001). 
Um estudo com 118 mulheres sujeitadas à intervenção de Le Fort com vigilância de variável (1 a 15 anos), explanou bons resultados anatômicos em relação ao pós operatório em 90,7\% dos casos, com uma recidiva total do prolapso apenas numa doente (GOLDMAN J, 1981).

Um estudo elaborado por Reisenauer C, et al. (2013), mostram que 58 mulheres tratadas com Le Fort colpocleise entre julho de 2007 e junho de 2011, 38 mulheres aceitaram participar do estudo e foram capazes de vir para uma visita de acompanhamento (66\%). 37 pacientes já tinham passado por um prolapso de órgão pélvico. O período de acompanhamento médio foi de 14 meses (oscilando de 3-41 meses). 16 mulheres tiveram a consulta de acompanhamento dentro de 1 ano pós-operatório, 12 entre 1 e 2 anos após a cirurgia e 10 pacientes mais de 2 anos de pós-operatório.

A média de idade dos pacientes na operação foi de 81,9 ( $\pm 6,4)$ anos (intervalo pacientes na operação foi de 81,9 $( \pm 6,4)$ anos (intervalo pacientes na operação foi de 81,9 $( \pm 6,4)$ anos (intervalo 63,9-95,9) e a média BMl foi de $24,5( \pm 3,4) \mathrm{kg} / \mathrm{m} 2$ (alcance $63,9-95,9)$ e a média BMl foi de $24,5( \pm 3,4) \mathrm{kg} / \mathrm{m} 2$ (alcance 63,9 95,9) e a média BMI foi de $24,5( \pm 3,4) \mathrm{kg} / \mathrm{m} 2$ (alcance $63,9-95,9)$ e a média BMI foi de $24,5( \pm 3,4) \mathrm{kg} / \mathrm{m}$ 2 (alcance 63,9-95,9) e a média BMI foi de $24,5( \pm 3,4) \mathrm{kg} / \mathrm{m} 2$ (alcance 16,4-31,1). 2 pacientes $(5 \%)$ não tiveram gravidez e $95 \%$ dos pacientes já tinham dado à luz um ou mais filhos. 19 mulheres (50\%) não passaram por cirurgia ginecológica anterior e 19 pacientes (50\%) já tinham passado por pelo menos uma operação ginecológica anterior. Vinte pacientes $(53 \%)$ eram tratadas pré-operatório com uma pessário para um ou mais anos. Anterior e reparação posterior foi realizada em 11 sujeitos $(29 \%)$, histerectomia vaginal em $9(24 \%)$, histerectomia abdominal em $7(18 \%)$, a suspensão ligamento sacro em $4(11 \%)$, vaginal malha reparação em $2(5 \%)$ e colposuspensão em um caso (3\%) anteriormente para o nosso procedimento colpocleise (REISENAUER C, 2013).

Em um relato do caso em (2001), foi descrito que uma paciente de idade de 84 anos, branca, viúva, XVI gesta, com prolapso uterino passou por um procedimento de colpocleise de intervento de exceção, que é desempenhado raramente, com indicações limitadas e precisas. É válido realçar que existe sempre a possibilidade de reincidência do prolapso, ou até mesmo ocorrer o aumento da gravidade da incontinência urinária ocasionada pela tração que é desempenhada sobre a parede vaginal antes e, ainda em muitos casos acarreta a formação de piométrio (KHOLI N, et al., 1996),

\section{Comparação Le Fort colpocleise com HV}

Denehy TR, et al. (2005), fazendo comparação Le Fort colpocleise (21 pacientes) com HV, anterior colporrafia, e colpoperineoplastia posterior (42 pacientes). Os pacientes operados por colpocleise eram mais velhos e o tempo de operação foi reduzida para metade. No grupo colpocleise observou que o prolapso ocorreu muitos tempo após a histerectomia. Parecia existir um curto espaço de anos entre a histerectomia e o prolapso no grupo de HV (14,7 anos) do que no grupo de histerectomia abdominal (20,4 anos), mas isso pode ser devido ao fato de que muitos dos pacientes HV já tinha alguma prolapso vaginal no momento da operação.

A cirurgia reconstrutiva corrige a vagina prolapsada visando restaurar parte da anatomia buscando sua normalidade, enquanto a cirurgia obliterativa corrige o prolapso extraindo ou fechando totalmente ou parcialmente o canal vaginal (colpocleose) afim de reduzir vísceras de volta à pelve Foi relatado que $1 \mathrm{em}$ cada 9 mulheres será submetida a cirurgia para incontinência ou prolapso aos 80 anos de idade. As taxas de recorrência a longo prazo de cirurgias reconstrutivas convencionais podem chegar a 50\%, com uma taxa de reoperação para falhas de até 30\% (WALTERS MD, et al., 2015; MIKOS T, 2016).

Estudos existentes na literatura são limitados com relação a sua qualidade. Relatos de casos permitem concluir que a colpocleise é um tratamento eficaz e duradouro para o prolapso genital, com taxas de sucesso de $91 \%$ a $100 \%$ (FITZGERALDMP, et al., 2006).

\section{Taxas de complicações}

Com relação às complicações desse procedimento, muitas são decorrentes da idade, como complicações cardíacas, pulmonares e vasculares, ocorrendo em aproximadamente $2 \%$ dos casos. Complicações maiores, 
como necessidade de transfusão e pielonefrite, são relatadas em aproximadamente $4 \%$ e normalmente estão associadas à histerectomia (HADDAD JM, 2015). Complicações menores como necessidade de UTI, hematoma vaginal, febre e tromboflebite, podem ocorrer em até $15 \%$ das mulheres operadas (FITZGERALD MP, et al., 2006). Recomenda a realização de colpocleise parcial, evitando manipulação na região uretral.

No estudo de Bochenska K, et al. (2017), foram identificadas 1.027 mulheres na base dedados que foi submetido a procedimentos de encerramento vaginais entre 2006 e 2014. A maioria dos pacientes (893, $87,0 \%)$ foram submetidos colpocleise sozinho, e o restante $(134,13,0 \%)$ foram submetidos a concomitante $\mathrm{VH}$. Estudos na área ginecológica têm apontado que o aumento dos tempos operativos está associado a complicações pós-operatórias em vaginal, laparoscópica, e histerectomia robótico (CATANZARITE T, et al., 2015). Ainda de acordo com o autor mesmo que as taxas de complicação apresentadas são baixas, quando ocorre a realização da HV juntamente com a colpocleise, existe um aumento expressivo quanto ao risco de complicações médicas graves, que é acometida isoladamente da faixa etária do paciente e aumento do tempo da operação. Esta comprovação sugere que HV não precisa ser desempenhada frequentemente no mesmo instante da colpocleise a exceto que exista uterino peculiar ou uma patologia do colo do útero que exige histerectomia.

A infecção é a complicação mais partilhada na HV, podendo variar de 1 a $5 \%$. Multifatores estão associados à infecção, dentre pode-se citar o: manuseio eminente dos tecidos, comorbidades que diminuem a imunidade, tempo cirúrgico estendido, vulvovaginites, e sobretudo a formação de hematomas (SEGEV Y, et al., 2010). As condições que elevam o risco de desencadear uma infecção pós-operatória incluem: idade avançada acima de 60 anos, casos de obesidade, quando é necessário transfusão sanguínea, faz- se o uso de tabagismo, sondagem vesical acima de $24 \mathrm{~h}$ e lesões intraoperatórias da bexiga e ureter (KJOLHEDE P, et al., 2011). Para concluir, de forma geral, é descrito em meio os principais benefícios e desvantagens que se envolvem com a execução de HV ou colpocleise de Le Fort em pacientes idosas com prolapso uterino total. Adorno SDS, et al. (2018), em seu estudo consegue relatar os benefícios e as desvantagens da HV tais como parte benéfica: demanda menor tempo operatório, minimamente invasivo, baixa morbidade e sem a existência de cicatriz visível, necessidade de menor tempo para o retorno às atividades cotidianas, baixa incidência de complicações intraoperatórias, ótima relação custo-benefício, menor perda sanguínea durante a operação, implica custos bem mais baixos para o Sistema de Saúde e por fim menor tempo de deficiência e hospitalização.

E as desvantagens estão correlacionadas com: incisão na vagina, histerectomias vaginais em pacientes sem prolapso genital, risco de quadro infeccioso em pacientes com sobrepeso ou em estado de obesidade, frequência de conversão da cirurgia de histerectomia de via vaginal para abdominal, possibilidade de lesão vesical, hematoma de cúpula, infecções e tromboembolismo, recorrente uso da laparoscopia para a cirurgia vaginal em casos complexos. Já outros estudos abordados revelam os benefícios e desvantagens da colpocleise a le fort, dentre os benefícios estão: realizado por via vaginal, redução do tempo da cirurgia representa menor risco cirúrgico, reduz o uso de medicações no pós-operatório, facilita a alta hospitalar.

\section{CONSIDERAÇÕES FINAIS}

Ainda que algumas mulheres portadoras de prolapso uterino completo tenham preferência de interromper a sua atividade sexual devido à sua condição clínica, muitas ainda procuram conseguir reiniciar a vida, mesmo aquelas com faixa etária elevada, buscando assim cada vez mais valorizar suas funções sexuais, sendo muito importante alcançar tudo o que é oferecido pelas técnicas cirúrgicas que tem como fundamento primordial proteger a anatomia e função vaginal, de forma que sejam escolhidas garantindo a segurança das pacientes e portadoras de comorbidades. As vantagens da HV reafirmam que nesse procedimento ocorre menor quantidade de perda sanguínea intra-operatória, menor intensidade de dor pós-operatória, menor presença de utilização de analgésicos e menor tempo de volta às atividades após a cirurgia. Le Fort colpocleise continua a ser uma excelente opção cirúrgica em pacientes bem selecionados. É importante que os cirurgiões pélvicos gestão prolapso genital são confortáveis com uma variedade de abordagens, incluindo ambas as cirurgias reconstrutivas e obliterante.

REAS/EJCH | Vol.Sup.n.47 | e3105 | DOI: https://doi.org/10.25248/reas.e3105.2020 Página 6 de 8 


\section{REFERÊNCIAS}

1. ADORNO SDS, et al. Vantagens da histerectomia vaginal comparado à histerectomia abdominal em mulheres sem prolapso genital. Revista Eletrônica Acervo Saúde. 2018; 17: e97.

2. BOCHENSKA K, et al .Complicações perioperatórias seguintes colpocleise com e sem histerectomia vaginal concomitante. Int Urogynecol J. 2017.

3. BOYD LR, et al. Effect of surgical volume on route of hysterectomy and short-termmorbidity. Obstet Gynecol.; 116(4):909-15, 2010.

4. CARRAMÃO S, et al. Estudo randômico da correção cirúrgica do prolapso uterino através de tela sintética de polipropileno tipo I comparando histerectomia versus preservação uterina. Rev. Col. Bras. Cir. vol.36 no.1 Rio de Janeiro Jan./Feb. 2009.

5. CATANZARITE T, et al. Mais tempo operatório durante laparoscópica benigna e histerectomia robótico está associada com um aumento de complicações peri-operatório de 30 dias. J Mínima invasiva Gynecol. $2015 ; 22$ (6): 1049 - 58.

6. COSTA AAR. Comparação dos resultados intra e pós-operatórios da histerectomia vaginal versus abdominal em mulheres sem prolapso genital, em um hospital-escola do Recife: ensaio clínico randomizado. [Dissertação de Mestrado]. IMIP, Recife, 2003.123 p.

7. DENEHY TR, et al. Modifed colpocleise parcial Le Fort com Kelly aplicação uretral e colpoperineoplastia posterior no medicamente comprometida idosos: uma comparação com histerectomia vaginal, anterior colporrafia, e colpoperineoplastia posterior. Am J Obstet Gynecol 173: 1.697-1.702, 1995.

8. FIGUEIREDO O, et al. Vaginal removal of the benign non prolapsed uterus: experience with 300 consecutive operations. Obstet Gynecol 1999; 94: 348-51.

9. FITZGERALD MP, et al. Colpocleisis: a review. Int Uro gynecol J. 2006,17: 261-271.

10. FITZGERALD MP, et al. Suporte pélvico, sintomas pélvicos e satisfação do paciente após colpocleise. Int Uro gynecol J Pelvic Pavimento Dysfunct. 2008; 19 (12): 1603 - 9.

11. GOLDMAN J, et al. The Neugebauer-Le Fort operation: a review of 118 partial colpocleisis. Eur J Obstet Gynecol Reprod biol. 1981;12:31-35.

12. HADDAD JM. Manual de uroginecologia e cirurgia vaginal. São Paulo: Federação Brasileira das Associações de Ginecologia e Obstetrícia (FEBRASGO), 2015.

13. HEISLER CA, et al. Effect of additional e constructive surgery on perioperative and post operative morbidity in women undergoing vaginal hysterectomy. ObstetGynecol.2009; 114(4):720-6.

14. HEISLER CA, et al. Improving quality of care: development of a risk-adjusted perioperative morbidity model for vaginal hysterectomy. Am J Obstet Gynecol. 2010; 202(2):e1- 3.

15. JACOBY VL, et al. Nationwide use of laparoscopic hysterectomy compared with abdominal and vaginal approaches. Obstetrics and gynecology. 2009;114(5):1041-8.

16. KHOLI N, et al. Pyometra following Le Fort colpocleisis. Int Urogynecol J PelvicFloorDysfunct1996;7:264-6.

17. KJOLHEDE P; et al. Vaginal cleansing and postoperative infectious morbidity in vaginal hysterectomy. A register study from the Swedish National Register for Gynecological Surgery. Acta Obstet Gynecol Scand.; 90(1):63-71, 2011.

18. KOVAC SR. Hysterectomy outcomes in patients with similar indications. Obstet Gynecol 2000; 95:787-93.

19. LIMA JM et al. Caracterização das mulheres idosas que realizaram histerectomia vaginal em um hospital universitário. Congresso Internacional de Envelhecimento humano: avanços das ciências e das políticas públicas para o envelhecimento, 2013.

20. MAHER CF, et al. Uterina preservação- ou histerectomia na colpopexiasacroespinhal para prolapso vaginal utero-? Int Urogynecol J Pelvic Pavimento Dysfunct. 2001;12: 381-384

21. MAHERCF, et al. Surgical management of pelvic organ prolapse in women. Cochrane Database Syst Rev 2007; 18(3):CD004014.

22. MARCUS CP, et al. Prolapso genital: via vaginal versus abdominal de reparação. Curr Opin Obstet Gynecol.2001; 13: 499-505.

23. MELO MCB; BARROS EN. Histerectomia e Simbolismo do útero: possíveis repercussões na sexualidade feminina. Revista Sociedade Brasileira de Psicologia Hospitalar, Rio de Janeiro, v.12, n.2, dez. 2009.

24. MENARD JP, et al. Prolapso genital de age efemme de 70 years mais: o revue literatura. E Ginecologia Obstetrícia fertilité 2008; 36: 67-73.

25. METTLER L, et al. Hysterectomy: current methods and alternatives [editorial]. Obstetrics e Gynecoly International. 2010; 20(10): 705073.

26. MIKOS T, et al. Enlightening the mechanisms of POP recurrence after Le Fort colpocleisis. Case report and review. Int Urogynecol J. 2016; 28: 971-978.

27. MINISTÉRIO DA SAÚDE. Proadess [homepage na internet]. Taxa de internacão para histerectomia em mulheres com 20 anos ou mais.2014.

28. NÍTIDO CC, et al. Imagem corporal, arrependimento e satisfação seguinte colpocleise. Am J ObstetGynecol. 2013; 209 (5): 473 e1 - 7.

29. PIAZZA MJ, et al. Histerectomia total versus histerectomia supracervical. FEMINA. Out. 2010;39(10):479-484 
30. SAMUELSSON EC, et al. Signs of genital prolapse in a Swedish population of women 20 to 59 years of age and possible related factors. Am J Obstet Gynecol. 1999; 180(2 Pt 1): 299-305.

31. SCHORGE JO, et al. Prolapso de órgão pélvico. In: Ginecologia do Williams, John O. Schorge, et al. tradução: Celeste Inthy, et al; revisão técnica: Suzana Pessini. Porto Alegre: Artmed; 2011; 532-5

32. SEGEV Y, et al. Symptomatic pelvic hematoma following transvaginal reconstructive pelvic surgery: incidence, clinical presentation, risk factors, and outcome. Eur J Obstet Gynecol Reprod Biol.; 2010; 153(2): 211-4.

33. SUTTON C. Hysterectomy: a historical perspective. Baillieres Clin Obstet Gynaecol.; 11(1):1-22, 1997.

34. WALLWIENER D. Atlas de cirurgia ginecológica. 7 ed. Artmed: São Paulo, 2012.

35. WALTERS MD, et al. Obliterative procedures for pelvic organ prolapse. In: Walters MD, Karram MM, editors. , eds. Urogynecology and Reconstructive Pelvic Surgery. Philadelphia, PA: Elsevier; 2015: 400-410.

36. WU JM, et al. Hysterectomy rates in the United States, 2003. Obstet Gynecol, 2007;110: 1091-1095. 